\title{
A Prospective Comparative Study of Unstable Inter Trochanteric Fracture by Proximal Femoral Nail and Proximal Femoral Nail Antirotation in Elderly Patients in Terms of Functional and Radiological Outcomes
}

\author{
Ahmer Hasmat ${ }^{1}$, Hetandar Kumar Bhartiya², Om Prakash³, Vyas Narayan Shukla ${ }^{4}$ \\ ${ }^{1}$ Department of Orthopaedics, MLN Medical College, Prayagraj, Uttar Pradesh, India. ${ }^{2}$ Department of Orthopaedics, \\ TMMC and Research Centre, Moradabad, Uttar Pradesh, India. ${ }^{3}$ Department of Orthopaedics, Surya Hospital, Amethi, \\ Uttar Pradesh, India. ${ }^{4}$ Department of Orthopaedics, MLN Medical College, Prayagraj, Uttar Pradesh, India.
}

\section{ABSTRACT}

\section{BACKGROUND}

Intramedullary devices allow for stable anatomical fixation of more comminuted fractures without shortening the abductor lever arm or changing the proximal femoral anatomy. Between intramedullary devices like proximal femoral nail and proximal femoral nail antirotation, the helical blade of latter is believed to provide stability, compression and rotational control of the fracture with higher cut out strength. The aim of the study was to manage and compare the functional and radiological outcomes between Proximal Femoral Nail or Proximal Femoral Nail Anti-rotation II in elderly patients of unstable intertrochanteric fractures.

\section{METHODS}

This is a prospective comparative study conducted in the Department of Orthopaedics at MLN Medical College and Swaroop Rani Hospital, Prayagraj, from November 2017 till June 2019.

\section{RESULTS}

Preoperative and post-operative clinical evaluation, radiological evaluation, assessment of operative time, number of fluoroscopy shoots, time for fracture union, and post-operative complications in all cases were noted. Results were evaluated using Harris hip score. There was significant $(p=0.0001)$ association of final outcome between the groups.

\section{CONCLUSIONS}

PFN A offers significant results over PFN in relation to post-operative complications. PFN A significantly reduces the operative time, amount of blood loss and fluoroscopic imaging as compared to PFN. However PFN A offers no significant benefits over PFN in terms of post-operative functional recovery or complications.
Corresponding Author: Hetandar Kumar Bhartiya, Moh. Sottiyan, Near Mai Ji Ka Mat, H. No. 20, Post-Bijnor-246701 District, Uttar Pradesh, India. E-mail: bhartiya.14@gmail.com

DOI: $10.14260 / \mathrm{jemds} / 2020 / 196$

Financial or Other Competing Interests: None.

How to Cite This Article:

Hasmat A, Bhartiya HK, Prakash O, et al. A prospective comparative study of unstable inter trochanteric fracture by proximal femoral nail and proximal femoral nail antirotation in elderly patients in terms of functional and radiological outcomes. J. Evolution Med. Dent. Sci. 2020;9(12):909912, DOI: 10.14260/jemds/2020/196

Submission 27-01-2020,

Peer Review 02-03-2020,

Acceptance 04-03-2020,

Published 23-03-2020.

\section{KEY WORDS}

Proximal Femur Nail, Proximal Femur Nail Antirotation, Intertrochanteric Fracture 


\section{BACKGROUND}

Intertrochanteric fracture of femur is a commonly encountered injury among people above 60 years of age. ${ }^{1}$ To restore the patients to pre-injury state at the earliest, the goal of treatment is stable fixation that promotes early postoperative mobilization. ${ }^{2}$ Intramedullary devices appear to be highly appropriate due to their biochemical properties. Proximal femoral nail with controllable sliding and short lever arm are associated with high complication rates of the neck and head screw. ${ }^{3}$ PFNA2 having helical blade rather than the screw for neck or head fragment that have reduced complication rates. ${ }^{3}$

In the present study, an effort was made to compare the functional and radiological outcomes of PFA2 over PFN in the management of unstable intertrochanteric fractures in elderly patients.

\section{METHODS}

This is a prospective non-randomized control trial study conducted in the Department of Orthopaedics at MLN Medical College, Prayagraj (U.P.) from November 2017 till June 2019. Sixty patients with unstable intertrochanteric fracture were selected for the study. The study sample was selected at conveniences. All data of the patients were collected in a preformed proforma manner and informed consent was also taken from patients and their attendants. The elderly patients with unstable intertrochanteric fracture that were attending MLN Medical College and Swaroop rani hospital were assessed pre-operatively and functional outcomes were evaluated postoperatively. A total of 30 patients were included in each group. There were 3 patient loss to follow up in proximal femoral nail group and 4 in Proximal Femoral Nail Anti-rotation. One patient died in proximal femoral nail group. Hence, 26 patients were analysed in each group

\section{Inclusion Criteria}

1. Age more than 60 years.

2. Unstable intertrochanteric fractures.

3. Acute unilateral intertrochanteric fractures belonging to AO 31-A2, AO 31-A3.

\section{Exclusion Criteria \\ 1. Pathological fractures. \\ 2. Open fracture. \\ 3. Any other fracture in same extremity. \\ 4. Neuromuscular disorders. \\ 5. Life threatening co-morbid conditions.}

All the patients were admitted in the hospital and a detailed history was taken emphasizing on the mode of injury and medical illness. Prior to the surgery an evaluation was done on cardiovascular, respiratory and abdominal system. Musculoskeletal system examination was done to rule out other bone and joint injuries. The limb attitude was noted for ecchymosis, deformity and shortening. Hip examination was carried out to look for any swelling, scar and bruising. Distal neurovascular status was checked.

\section{Surgical Procedure}

Proximal Femoral Nail Group

All the patients received spinal anaesthesia and was placed supine on the fracture table. The operative limb was kept on traction and the opposite limb kept in abduction and flexion to give space to the C-Arm. Reduction achieved through the traction and internal rotation primarily and abduction or adduction as required and confirmed by using C-Arm by AP and the lateral view. Lateral incision of $2-3 \mathrm{~cm}$ was made 2 $\mathrm{cm}$ superior to the apex of greater trochanter. The apex of the GT was exposed by bluntly dissecting gluteus medius. Entry point was taken with awl. A guide wire of $2.8 \mathrm{~mm}$ was inserted into the shaft of femur and reaming of the proximal femur was done. Nail was fixed on the jig and was inserted in the femur. Through the jig and the drill sleeve, guide wires for the screws were inserted. In antero-posterior view the ideal position of the guide wires were parallel to each other and in the distal $1 / 2$ of the neck and in the lateral view it was in a single line in the Centre of the neck. The guide pins were inserted up to $5 \mathrm{~mm}$ from the articular surface of the femoral head and size of the lag screw was determined. In the distal part of the nail 1 or 2 static or dynamic $4.9 \mathrm{~mm}$ interlocking bolts were inserted.

\section{Proximal Femoral Nail Anti-Rotation Group}

All the patients received spinal anaesthesia and was placed supine on the fracture table. The operative limb was kept on traction and the opposite limb kept in abduction and flexion to give space to the C-Arm. Reduction achieved through the traction and internal rotation primarily and abduction or adduction as required and confirmed by using C-Arm by AP and the lateral view. PFNA2 was inserted similar to conventional proximal femoral nail except a single pin was driven into the neck of femur from the proximal locking hole with matching guide. Antero-posterior view of image intensifier showed that the pin was $5-10 \mathrm{~mm}$ away from the articular surface of femoral head. The lateral view of image intensifier showed that it was located at the centre of or slightly posterior to the head of femur. The reamer was used to drill the neck to make room for helical blade along the pin and final position of the blade was checked under image intensifier. In the distal part of the nail 1 or 2 static or dynamic $4.9 \mathrm{~mm}$ interlocking bolts were placed.

Intraoperatively the duration of surgery and number of images shot on the C-Arm were recorded. Third-generation cephalosporin was being continued for 3 days. Anteroposterior (AP) and lateral radiograph was taken for all the patients.

\section{Physiotherapy}

Quadriceps (Strengthening) exercises and pumping of the calf were initiated as early as the patient was out of anaesthesia, followed by mobilization of the knee and ankle on day 1 . Touchdown weight bearing was started from day 3 of the surgery by using a walking frame up to 6 weeks, followed by gradual weight bearing depending on the fracture union.

\section{Follow Up/Radiological}

Antero-posterior (AP) and the lateral radiograph was taken at each follow-up for the evaluation of fracture union and 
position of the implant. At 6 weeks, 3 months, 6 months and 9 months, all patients are clinically and radiologically evaluated for union of the fracture and complications.

\section{Functional Evaluation}

Functional evaluation was done at 6 weeks, 3 months, 6 months and 9 months at each follow up using Harris Hip Score. ${ }^{4}$

\section{Statistical Analysis}

SPSS version 23 was used for statistical analysis. All study variables were represented using frequency and percentage. Chi-square test/unpaired- $t$ test was used to compare the study variables between the study groups. The P-value less than $(\mathrm{p}<0.05)$ was taken as significant.

\section{RESULTS}

Table- 1 shows the patients distribution according to the age between the groups. More than one third of patients of Proximal femoral nail group (46.2\%) and $73.1 \%$ of Proximal Femoral Nail Anti-rotation were between $61-70$ years. The mean age of patients of PFN group and PFNA was $71.47 \pm 4.16$ and $70.17 \pm 8.96$ years respectively. The age difference between the groups was not significant $(p>0.05)$ showing the comparability of the groups in terms of age. Table- 2 shows the patients distribution according to the gender between the groups. More than half of patients of Proximal femoral nail group (57.7\%) and $46.2 \%$ of Proximal Femoral Nail Antirotation were males. The gender difference between the groups was not significant $(p>0.05)$ showing the comparability of the groups in terms of gender. Table 3 shows the comparison of operating time between the groups. The operating time was significantly $(\mathrm{p}=0.0001)$ higher in patients of Proximal femoral nail group $(84.23 \pm 9.92)$ than Proximal Femoral Nail Anti-rotation (61.35 \pm 6.03 ). Table-4 shows the comparison of no. of C-Arm shoot between the groups. The mean number of C-Arm shoot was significantly $(\mathrm{p}=0.0001)$ higher in PFN $(34.65 \pm 3.13)$ group than PFNA (29.46 \pm 1.90$)$. Table-5 shows the comparison of Harris hip score between the groups across the time periods. There was no significance $(p>0.05)$ in HHS between the groups across the time period.

Screw back-out was in $3.84 \%$ patients of PFN and in $3.84 \%$ of PFNA. However, Z-effect was in $7.69 \%$ of PFN and nil in PFNA group as shown in table-7 Table-8 shows the comparison of complications between the groups. The complication was present in $11.5 \%$ of proximal femoral nail group and in 3.8\% of Proximal Femoral Nail Anti-rotation. There was significant association of complications between the groups. Table- 9 shows the comparison of final results between the groups. Excellent outcome was in 50\% of proximal femoral nail group and in $69.2 \%$ of Proximal Femoral Nail Anti-rotation. There was significant $(\mathrm{p}<0.05)$ association of final outcome between the groups.

\begin{tabular}{|c|c|c|c|}
\hline Gender & PFN (n=26) & PFNA $(n=26)$ & $\mathbf{p}$ \\
\hline Male & 16 & 14 & \multirow{2}{*}{0.40} \\
\hline Female & 10 & 12 & \\
\hline \multicolumn{4}{|c|}{ Table 1. Gender Distribution } \\
\hline
\end{tabular}

\begin{tabular}{|cccc|}
\hline Time Periods & PFN & PFNA & p \\
6 weeks & $26.11 \pm 4.90$ & $28.38 \pm 5.31$ & 0.11 \\
3 months & $46.42 \pm 5.50$ & $46.50 \pm 8.46$ & 0.96 \\
6 months & $69.65 \pm 7.57$ & $67.42 \pm 8.53$ & 0.32 \\
9 months & $88.03 \pm 8.46$ & $89.36 \pm 11.18$ & 0.63 \\
\hline \multicolumn{4}{|r}{ Table 2. Comparison of Harris Hip Score between } \\
& the Groups across the Time Periods \\
\hline Unpaired t-test & & \\
\hline
\end{tabular}

\begin{tabular}{|ccccccc|}
\hline $\begin{array}{c}\text { Time } \\
\text { Periods }\end{array}$ & & $\begin{array}{c}\text { PFN } \\
\text { (n=26) }\end{array}$ & \multicolumn{5}{c|}{$\begin{array}{c}\text { PFNA } \\
\text { (n=26) }\end{array}$} & p \\
6 weeks & Callus present & 26 & $100.0 \%$ & 26 & $100.00 \%$ & - \\
3 months & Union in progress & 26 & $100.0 \%$ & 26 & $100.00 \%$ & - \\
6 months & Union in progress & 26 & $100.0 \%$ & 26 & $100.00 \%$ & - \\
& Union in progress & 3 & $\%$ & 0 & $0.0 \%$ & 0.84 \\
\cline { 2 - 3 } & Union present & 23 & $\%$ & 25 & $96.11 \%$ & \\
\hline & Table 3. Comparison of Radiological Outcome & \\
& between the Groups across the Time Periods \\
\hline
\end{tabular}

\begin{tabular}{|ccccc|}
\hline Complications & \multicolumn{2}{c|}{ PFN (n=26) } & \multicolumn{2}{c|}{ PFNA (n=26) } \\
Screw backout & 1 & $3.84 \%$ & 1 & $3.84 \%$ \\
Z-EFFECT & 2 & $7.69 \%$ & 0 & $0 \%$ \\
\hline \multicolumn{3}{|c}{ Table 7. Post-Operative Complications } \\
\hline
\end{tabular}

\begin{tabular}{|cccccc|}
\hline Complications & \multicolumn{3}{c|}{ PFN } & $(\mathbf{n = 2 6 )}$ & \multicolumn{2}{c|}{ PFNA (n=26) } & p \\
Present & 3 & $11.5 \%$ & 1 & $3.8 \%$ & $0.04^{*}$ \\
Absent & 23 & $88.5 \%$ & 25 & $96.2 \%$ & \\
\hline Table 8. Comparison & of Complications between the Groups \\
\hline
\end{tabular}

\begin{tabular}{|cccccc|}
\hline Results & \multicolumn{1}{c|}{ PFN (n=26) } & \multicolumn{3}{c|}{ PFNA (n=26) } & p \\
Excellent & 13 & $50.0 \%$ & 18 & $69.2 \%$ & \\
Good & 9 & $34.6 \%$ & 2 & $7.7 \%$ & $0.0001^{*}$ \\
Fair & 1 & $3.8 \%$ & 3 & $11.5 \%$ & \\
Poor & 3 & $11.5 \%$ & 3 & $11.5 \%$ & \\
\hline \multicolumn{5}{r}{ Table 9. Comparison of Final Results between the Groups } \\
\hline
\end{tabular}

\section{DISCUSSION}

In the present study, the mean age of patients of PFN group and PFNA was $71.47 \pm 7.72$ and $70.17 \pm 8.96$ years respectively. The age difference between the groups was not significant $(p>0.05)$. However, a significant $(p=0.05)$ difference in the age was reported between patients treated with PFN (60.78 years) and PFNA (74.12 years) (Sharma et al, 2017). ${ }^{5}$ PFN group (57.7\%) and $46.2 \%$ of PFNA were males in this study. In the study by Sharma et al (2017), there were $69.5 \%$ males in PFN group and $40 \%$ in PFNA group. 5 There was no significance $(p>0.05)$ in Harris hip score between the groups across the time periods in patients of PFNA than PFN. Kashid et al (2016) found that the mean Harris hip score of PFNA group was comparably greater than the PFN group although there was no significant ( $p=0.562$ ) difference as in the present study. ${ }^{6}$

In our research operation time was significantly ( $\mathrm{p}=0.0001)$ higher in PFN than (84.23 \pm 9.92 minutes) PFNA (61.35 \pm 6.03 minutes). Kashid et al (2016) also reported similar finding with this study in which the mean operative time was significantly lower in PFNA group than the PFN group $(35.20 \pm 6.03$ minutes vs. $43.32 \pm 8.20$ minutes, $(p$ $<0.001) .{ }^{6}$ In the present study, the mean number of images shoot was significantly $(\mathrm{p}=0.0001)$ higher in PFN $(34.65 \pm 3.13)$ group than PFNA $(29.46 \pm 1.90)$. This finding is in agreement with the study by Kashid et al (2016) in which 
the mean number of images shoots per-operatively was significantly less inPFNA group than the PFN group $\left(18.60 \pm 3.12\right.$ vs $29.52 \pm 4.85(\mathrm{p}<0.001){ }^{6}$ This study noted post-operative complications in $3(11.5 \%)$ cases of PFN and 1 (3.8\%) case of PFNA. Mohan et al (2016) reported that there were 2 cases of superficial infection in PFNA and 2 each of superficial\& deep infection in PFN 7 . Sharma et al (2017) reported higher complications in both PFN (34.7\%) and PFNA (12\%) than the present study. ${ }^{5}$

Park et al (2010) found significantly better outcomes in terms of functional scores and the mobility scores and less rate of complications with helical blade nails. In this study, screw back-out was in 3.84\% patients of PFN and in 3.84\% of PFNA. However, z effect was in $7.69 \%$ of PFN and nil in PFNA group. Sharma et al (2017) reported that screw back-out and z-effect was in $28.4 \%$ and $14.2 \%$ patients of PFN respectively and both were nil in PFNA group. ${ }^{5}$ In this study, excellent outcome was in $50 \%$ of PFN group and in $69.2 \%$ of PFNA. In the study by Mohan et al (2016), in PFNA group, excellent outcomes was shown in 45 cases, whereas in the PFN group, excellent in 37 cases. $^{7}$

\section{CONCLUSIONS}

This study suggests that there is no difference in functional outcomes with both the implants; however, post-operative complications are less with PFNA2 as compared to PFN. Duration of surgery, amount of blood loss and fluoroscopic imaging also reduces significantly with the use of PFNA2 as compared to PFN.

\section{REFERENCES}

[1] MaheshKumar NB, Mahesh U, SantoshKumar S. A comparative study of proximal femoral fracture fixation with proximal femoral nail and Dynamic hip screw \& plating. International Journal of Orthopaedics 2017;3(1):499-505.

[2] Hussain N, Patel HB, Patil ND. Management of complex intertrochanteric fracture of femur in elderly patientsdynamic hip screws or proximal femoral nails or arthroplasty. Int J Res Orthopedics 2017;3(4):656-60.

[3] Park JH, Lee YS, Park JW, et al. A comparative study of screw and helical proximal femoral nails for the treatment of intertrochanteric fractures. Orthopedics 2010;33(2);81-5.

[4] Harris WH. Traumatic arthritis of the hip after dislocation and acetabular fractures: treatment by mold arthroplasty: an end-result study using a new method of result evaluation. J Bone Joint Surg 1969;51(4):737-55.

[5] Sharma A, Mahajan A, John B. A comparison of the clinicoradiological outcomes with proximal femoral nail (PFN) and proximal femoral nail antirotation (PFNA) in fixation of unstable intertrochanteric fractures. Journal of Clinical and Diagnostic Research: JCDR 2017;11(7):RC05-RC09.

[6] Kashid MR, Gogia T, Prabhakara A, et al. Comparative study between proximal femoral nail and proximal femoral nail antirotation in management of unstable trochanteric fractures. Int J Res Orthop 2016;2(4):354-8.

[7] Mohan NS, Shivaprakash SU. PFNA v/s PFN in the management of unstable intertrochanteric fractures. Journal of Evolution of Medical and Dental Sciences 2015;4(24):4086-92. 